\title{
Immobilized iron Schiff base histidine complexes on Al-MCM-41 and zeolite $Y$ as catalyst for oxidation of cycloalkanes
}

\author{
Faezeh Farzaneh • Samaneh Sohrabi • \\ Mina Ghiasi · Mehdi Ghandi • Vahid Daadmehr
}

(C) Springer Science+Business Media, LLC 2012

\begin{abstract}
Iron complexes of $N$-salicylidene-L-histidine with or without bipyridine ligand immobilized on Al-MCM41 and zeolite $\mathrm{Y}$ designated as $\mathrm{Fe}($ sal-L-his)(bipy)complex/ Al-MCM-41 or Fe(sal-L-his)complex/Al-MCM-41 and Fe (sal-L-his)(bpy)complex/Y or Fe(sal-L-his)complex/Y respectively, were prepared and characterized by $\mathrm{X}$-ray powder diffraction (XRD), FT-IR, $\mathrm{N}_{2}$ adsorption/desorption and chemical analysis techniques. Fe(sal-L-his)/Al-MCM-41 and $\mathrm{Fe}$ (sal-L-his)(bipy)complex/Al-MCM-41 were found to successfully catalyze the oxidation of cyclohexane, methyl cyclohexane, cyclooctane and adamantane with $\mathrm{H}_{2} \mathrm{O}_{2}$. The oxidation results and promising catalytic behavior of $\mathrm{Fe}$ (salL-his)(bipy)complex/Al-MCM-41 for oxidation of cyclooctane with $90 \%$ conversion and excellent selectivity toward the formation of cyclooctanone will be discussed in this presentation.
\end{abstract}

Keywords Iron Schiff base complex $\cdot$ Al-MCM-41 . Oxidation catalyst $\cdot$ Cycloalkanes

Electronic supplementary material The online version of this article (doi:10.1007/s10934-012-9596-8) contains supplementary material, which is available to authorized users.

F. Farzaneh $(\bowtie) \cdot$ S. Sohrabi · M. Ghiasi

Department of Chemistry, University of Alzahra,

Vanak, Tehran, Iran

e-mail: faezeh_farzaneh@yahoo.com; farzaneh@alzahra.ac.ir

M. Ghandi

School of Chemistry, College of Science,

University of Tehran, Tehran, Iran

V. Daadmehr

Magnet and Superconducting Research Lab, Department

of Physics, University of Alzahra, Vanak, Tehran, Iran

\section{Introduction}

The catalytic oxidation of organic substrates by transition metal complexes is an area of current interest [1]. Transition metal complexes with porphyrin, phthalocyanine and Schiff base ligands have been utilized for oxidation of various hydrocarbons [2-5]. Schiff base ligands are ubiquitous in coordination chemistry where they are used in the synthesis of a large variety of transition metal complexes with a range of structural architectures [6-9]. Among the various metals, iron is the most abundant metal in nature and is indispensable species of nearly all organisms. Nature has developed a number of metaloenzymes such as heme containing as the Cytochrome P-450 and non-heme monooxygenases $[10,11]$. There has been significant interest in modeling such enzyme active sites and developing the biomimetic alkane hydroxylation catalyst $[12,13]$. In spite of the fact that many investigations and research have been performed on synthesis and biomimetic oxidation chemistry of iron complexes, the design of new iron Schiff base complexes as oxidation catalysts in organic transformations is the subject of recent research [14-17].

Utilization of solid supported liquid phase catalysts (SLPC) would be attractive since they offer the advantages of easy catalyst separation, possible catalyst recycle and sometimes high activity and selectivity [18]. The immobilization of metal complexes within solid supports such as microporous (zeolites) or mesoporous (MCMs) has proven to be an important step in heterogeneous enzymatic catalysis [19-22]. Iron complexes with many type of ligands such as porphyrins, salens, and phthalocyanines have been used for the selective oxidation of hydrocarbons [19-27].

In this study, attempts has been made to immobilize iron (III) complexes of $\mathrm{N}$-salicylidene-L-histidine designated as $\left[\mathrm{Fe}\right.$ (sal-L-his) $\left.\mathrm{Cl} \bullet \mathrm{H}_{2} \mathrm{O}\right]$ and $\mathrm{Fe}[($ sal-L-his)(bpy)Cl] within 
Al-MCM-41 and zeolite $\mathrm{Y}$ in order to investigate their catalytic activity on cyclohexane, methyl-cyclohexane, cyclooctane and adamantane oxidations with hydrogen peroxide as oxidant.

\section{Experimental}

\subsection{Materials}

All materials were of commercial reagent grade. Iron (III) chloride, methylamine, hexadecyltrimethylammoniumbromide (HDTMABr), tetraethylorthosilicate (TEOS), aluminum hydroxide, 2,2'-bipyridine (bpy), acetone, diethyl ether, salicylaldehyde, L-histidine, sodium acetate, ethanol, acetonitrile, $\mathrm{H}_{2} \mathrm{O}_{2}(30 \%)$, cyclohexane, methylcyclohexane, cyclooctane and adamantane were obtained from Merck Chemical Company and used without further purification. Zeolite Y as powder was purchased from Spag Company.

\subsection{Synthesis of Al-MCM-41}

The Al-MCM-41 ( $\mathrm{Si} / \mathrm{Al}=50)$ with molar composition of $1.00 \mathrm{SiO}_{2}$ : $1.60 \mathrm{MA}: 0.215$ HDTMABr: $125 \mathrm{H}_{2} \mathrm{O}: 0.02$ $\mathrm{Al}_{2} \mathrm{O}_{3}$ was prepared according to the reported method [28]. First, methylamine $(2.08 \mathrm{~mL})$ was added to distilled water (42 $\mathrm{mL}$ ) and the mixture was stirred at room temperature for $10 \mathrm{~min}$. HDTMABr (1.47 g) was gradually added to the above solution under stirring for $60 \mathrm{~min}$, followed by dropwise addition of TEOS $(2.1 \mathrm{~g})$. Aluminum hydroxide $(0.06 \mathrm{~g})$ in water $(5 \mathrm{~mL})$ was then added and the $\mathrm{pH}$ of the reaction mixture was adjusted to 8.5 by slow addition of hydrochloric acid solution ( $1 \mathrm{M}$ ). At this stage, a precipitate was formed. After $2 \mathrm{~h}$, the precipitate was separated and washed by centrifugation. The sample was dried at $45^{\circ} \mathrm{C}$ for $12 \mathrm{~h}$. A white powder was finally obtained after calcinations of the solid at $550{ }^{\circ} \mathrm{C}$ for $5 \mathrm{~h}$ to decompose the surfactant.

\subsection{Preparation of $\left[\mathrm{Fe}(\right.$ sal-L-his $\left.) \mathrm{Cl} \bullet \mathrm{H}_{2} \mathrm{O}\right]$}

$\mathrm{Fe}$ (sal-L-his) $\mathrm{Cl} \bullet \mathrm{H}_{2} \mathrm{O}$ was prepared according to the modified reported method [29]. Salicylaldehyde $(0.010 \mathrm{~mol})$ in ethanol $(20 \mathrm{~mL})$ was introduced into histidine $(0.010 \mathrm{~mol})$ dissolved in water $(20 \mathrm{~mL})$, followed by addition of sodium acetate solution $(0.020 \mathrm{~mol}$ in $10 \mathrm{~mL}$ water $)$. The color of the solution changed to deep yellow. An aqueous solution of $\mathrm{FeCl}_{3}(8.5 \mathrm{mmol}$, in $10 \mathrm{~mL}$ water $)$ was then slowly added to the mixture, while the solution was stirring. The resultant dark red solution was concentrated slowly using a rotary evaporator to obtain a precipitate. The precipitate was separated and washed with acetone and diethyl ether, and then dried at room temperature. $\mathrm{CHN}$ elemental analysis calculated (\%) for $\left[\mathrm{FeC}_{13} \mathrm{H}_{11} \mathrm{~N}_{3} \mathrm{O}_{3} \mathrm{Cl} \bullet \mathrm{H}_{2} \mathrm{O}\right]$ : $\mathrm{C} 42.59, \mathrm{H} 3.57, \mathrm{~N}$
11.46; Found: C 42.40, H 4.02, N 11.43. The FT-IR and $\mathrm{UV}-\mathrm{vis}$ spectra of the solid containing peaks respectively at $3,414,3,016,1,625,1,443,1,201,553,455 \mathrm{~cm}^{-1}$ and $\lambda_{\max }$ at $216,235,255,466 \mathrm{~nm}$ were identical to those of reported previously [29-31].

\subsection{Preparation of [Fe(sal-L-his)(bpy)Cl]}

Based on the reported method [29], $\mathrm{Fe}$ (sal-L-his)(bpy)Cl complex was prepared by addition of 2,2-bipyridyl (0.01 mol in $20 \mathrm{~mL}$ ethanol) to the $\mathrm{Fe}$ (sal-L-his)Cl complex $(0.01 \mathrm{mmol})$ and stirring the solution for $2 \mathrm{~h}$. After evaporation of the solvent, acetone was added to the residue and the product was collected by filtration and then drying under vacuum. $\mathrm{CHN}$ elemental analysis calculated (\%) for [ $\left.\mathrm{FeC}_{23} \mathrm{H}_{19} \mathrm{~N}_{5} \mathrm{O}_{3} \mathrm{Cl}\right]$ : $\mathrm{C}$ 54.71, $\mathrm{H}$ 3.80, N 13.23; Found: $\mathrm{C}$ 54.62, H3.65, N 13.33. The FT-IR and UV-vis spectra of the solid containing peaks respectively at 3,417, 3,024, 1,626, $1,443,1,195,538,456 \mathrm{~cm}^{-1}$ and $\lambda_{\max }$ at 204, 236, 288, 316, $484 \mathrm{~nm}[32,33]$ were identical to those of reported previously.

\subsection{Preparation of catalyst}

Al-MCM-41 (0.5 g) was added to the complex $(0.1 \mathrm{~g}$, $0.27 \mathrm{mmol}$ in $20 \mathrm{~mL}$ ethanol). The mixture was refluxed while stirring for $6 \mathrm{~h}$. The solid was filtered, washed with ethanol, soxhelet extracted with ethanol in order to remove the unimmobilizd complex followed by drying at room temperature. The same procedure was used for immobilization within zeolite $\mathrm{Y}$.

\subsection{Oxidation reactions}

Oxidation reactions were carried out at atmospheric pressure under reflux conditions. Typically, immobilized iron complex/Al-MCM-41 (or iron complex/Y) as catalyst $(0.1 \mathrm{~g}$ ) was added into the reaction flask containing cyclohexane (20 mmol dissolved in $5 \mathrm{~mL}$ acetonitrile) with slow stirring. After a few minutes, $\mathrm{H}_{2} \mathrm{O}_{2}(24 \mathrm{mmol}, 30 \%$ aqueous solution) was added and the mixture was refluxed for $6 \mathrm{~h}$. The solid was filtered and washed with fresh acetonitrile. The filtrate was then subjected to GC and GC Mass analysis.

\subsection{Characterization}

The FT-IR spectra of the samples were recorded using Bruker Tensor 27 IR spectrometer in $\mathrm{KBr}$ pellets over the range of 4,000-400 $\mathrm{cm}^{-1}$ under the atmospheric conditions. The UV-vis spectra were recorded on a UV-vis Perkin Elmer Lambda 35 spectrophotometer. The amount of iron complexes immobilized within of Al-MCM-41 or zeolite Y was determined by atomic absorption spectroscopy (AAS) 
with a GBC spectrophotometer using flame approach, after $\mathrm{HF}$ acid dissolution. BET surface areas and pore size distributions were determined by $\mathrm{N}_{2}$ adsorption/desorption at liquid nitrogen temperature $(77 \mathrm{~K}$ ) using Quanta chrome Nova version 2.2 Analyzer. Prior to adsorption/desorption measurement, the samples were equilibrated by degassing at $110{ }^{\circ} \mathrm{C}$ for $3 \mathrm{~h}$. Powder X-ray diffraction (XRD) measurements were performed on a scintillation diffractometer using $\mathrm{Cu} \mathrm{K} \alpha$ radiation $(\lambda=1.5406 \AA)$ at $40 \mathrm{kV}, 40 \mathrm{~mA}$. Oxidation products were analyzed by a GC and GC Mass model using an Agilent 6890 Series with FID detector, HP-5, $5 \%$ phenylmethylsiloxane capillary and an Agilent 5973 Network, mass selective detector, HP-5 MS 6989 Network GC system, respectively.

\section{Results and discussion}

\subsection{Synthesis and identification of catalysts}

Condensation of salicylaldehyde and histidine followed by treatment with $\mathrm{FeCl}_{3} \cdot 3 \quad \mathrm{H}_{2} \mathrm{O}$ under aerobic conditions afforded the desired iron $\mathrm{Fe}$ (sal-L-his) $\mathrm{Cl} \bullet \mathrm{H}_{2} \mathrm{O}$ complex (compound I, Scheme 1). As seen in the Scheme 1, compound II is obtained after addition of bipy ligand to the compound I solution. The FT-IR, UV-vis and chemical analysis of I and II were consistent with those of the authentic samples [29]. The structure of the complexes has been proposed on the basis of those provided previously [29]. To explore the magnetic properties, computation and optimization of energies and geometries of $\mathrm{Fe}\left(\right.$ sal-L-his) $\mathrm{Cl} \bullet \mathrm{H}_{2} \mathrm{O}$ and $\mathrm{Fe}$ (sal-L-his)(bpy)Cl complexes in doublet, quartet and sextet spin states were also carried out (see the supporting information). It was found that the sextet state for former complex, is 8.9 and $2.5 \mathrm{kcal} / \mathrm{mol}$ more stable than the doublet and quartet spin states, respectively. The results of the calculations for the latter complex showed that the intermediate spin state is 2.9 and $7.2 \mathrm{kcal} / \mathrm{mol}$ more stable than the high and low spin states in the solvent, respectively. Notably, results obtained through theoretical calculations (Kapa 5.0 and $2.8 \mathrm{emu} / \mathrm{gr} \mathrm{G} 10^{-5}$ ) are in agreement with magnetic properties of the prepared complexes.

\subsection{XRD studies}

The XRD patterns of Al-MCM-41, Fe(sal-L-his)complex/ Al-MCM-41 and Fe(sal-L-his)(bipy)complex/Al-MCM-41 are exhibited in Fig. 1a-c. The presence of a strong peak at $2 \Theta=1.7$ for the (100) reflection is consistent with that reported before [28]. All reflections can be indexed on a hexagonal lattice. No evidence of collapsed Al-MCM-41 structure is observed with the exception of reduction in the peak intensities due to complex immobilization [26, 34].

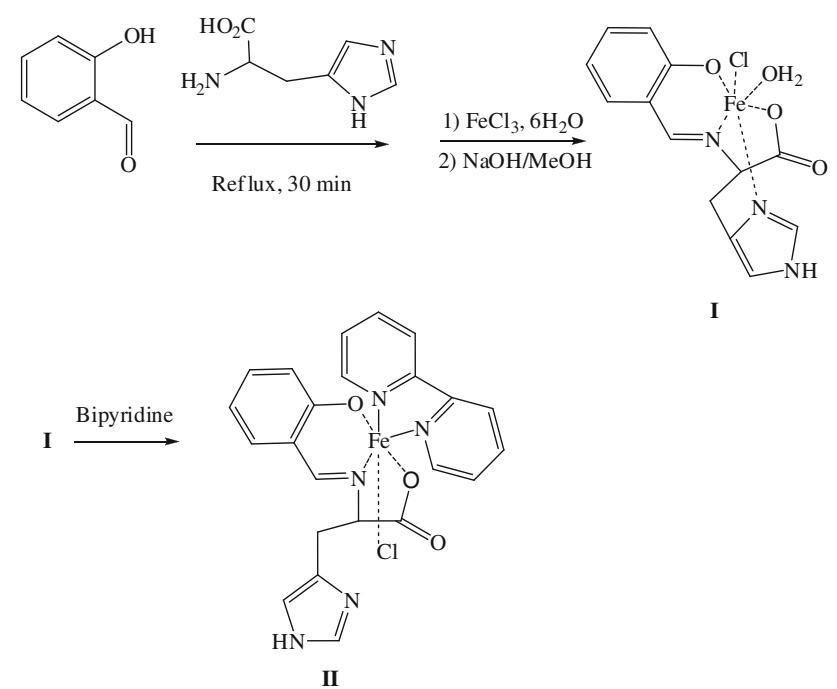

Scheme 1 Preparation of $\mathrm{Fe}\left(\right.$ sal-L-his)Cl $\bullet \mathrm{H}_{2} \mathrm{O}$ (I) and $\mathrm{Fe}$ (sal-Lhis)(bpy)Cl(II)

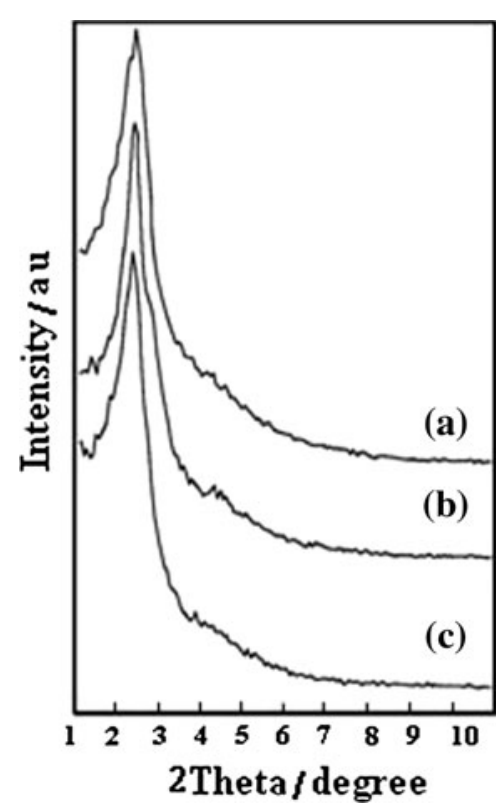

Fig. 1 X-ray diffraction patterns of $a$ calcined Al-MCM-41, $b$ Fe(sal-Lhis)complex/Al-MCM-41, $c$ Fe(sal-L-his)(bpy)complex/Al-MCM-41

The powder X-ray diffraction patterns of zeolite $\mathrm{Y}$ and $\mathrm{Fe}$ (sal-L-his)complex/zeolite $\mathrm{Y}$ recorded at $2 \Theta$ values between $5^{\circ}$ and $50^{\circ}$ are presented in Fig. 2a-c. Although the XRD patterns of zeolite $\mathrm{Y}, \mathrm{Fe}$ (sal-L-his)/zeolite $\mathrm{Y}$ and $\mathrm{Fe}$ (sal-L-his)(bipy)/zeolite $\mathrm{Y}$ are very similar but the intensities are weaker in the immobilized complexes (Fig. 2b, c). These observations indicate that the zeolite framework has not undergone a significant structural change during the complex immobilization. 


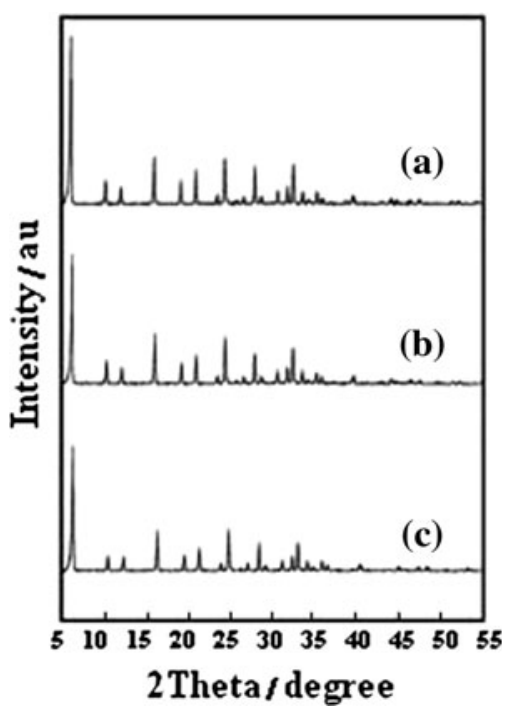

Fig. $2 \mathrm{X}$-ray diffraction patterns of $a$ zeolite $\mathrm{Y}, b \mathrm{Fe}$ (sal-L-his)complex/zeolite Y, $c$ Fe(sal-L-his)(bpy)complex/zeolite Y

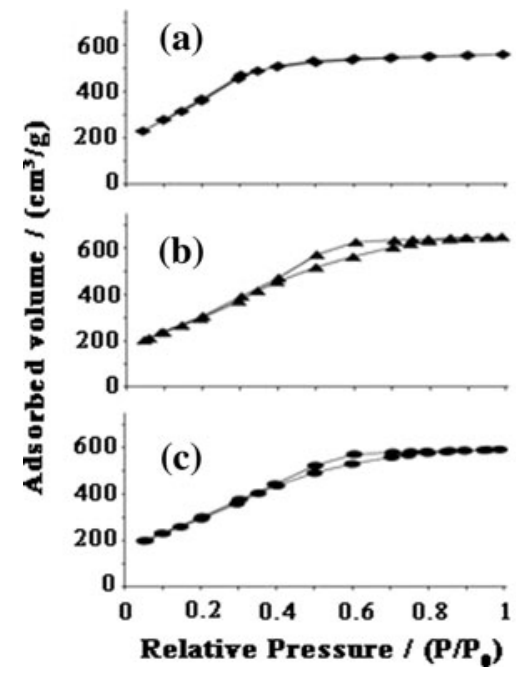

Fig. $3 \mathrm{~N}_{2}$ adsorption/desorption isotherms of $a$ Al-MCM-4, $b$ Fe(sal-Lhis)complex/Al-MCM-41 and $c$ Fe(sal-L-his)(bpy)complex/Al-MCM-41

\section{3 $\mathrm{N}_{2}$ adsorption/desorption studies}

The $\mathrm{N}_{2}$ adsorption/desorption isotherms of Al-MCM-41, Fe (sal-L-his)complex/Al-MCM-41and Fe(sal-L-his)(bipy)complex/Al-MCM-41 are shown in Fig. 3a-c. Three well-defined stages may be identified: (1) a slow increase in nitrogen uptake at low relative pressure corresponding to monolayermultilayer adsorption on the pore walls; (2) a sharp step at intermediate relative pressures indicative of capillary condensation within mesopores; (3) a plateau with a slight inclination at high relative pressures associated with multiplayer adsorption on the external surface of the crystals [3236]. Sharp inflections at $0.2-0.4$ for Al-MCM- 41 and $0.4-0.7$ for immobilized iron complexes are related to the capillary condensation and confirm the existence of uniform pores [32-36]. Complex intercalated Al-MCM-41 shows lower surface area in comparison to that of the neat Al-MCM- 41 . The increases observed in pore diameter and lattice parameters may have arisen due to the immobilized complexes within the Al-MCM-41. The same results are obtained for zeolite Y. The iron content percentages of the immobilized complexes are presented in Tables 1,2, respectively.

\subsection{FTIR studies of immobilized iron complexes within Al-MCM-41}

The FT-IR spectra of Al-MCM-41, Fe(sal-L-his)complex/ Al-MCM-41 and Fe(sal-L-his)(bpy)complex/Al-MCM-41 are given in Table 3. The broad bands appearing at 3,448, 3,446 and $3,432 \mathrm{~cm}^{-1}$ may be attributed to the surface silanol and-OH groups of adsorbed water, respectively. Some weak peaks appear at the regions of 2,987-2,900 $\mathrm{cm}^{-1}$ due to $\mathrm{C}-\mathrm{H}$ and $\mathrm{N}-\mathrm{H}$ vibrations of immobilized complex within Al-MCM-41. The bands at $1,099-1,075 \mathrm{~cm}^{-1}$ are due to the asymmetric stretching vibrations of $\mathrm{Si}-\mathrm{O}-\mathrm{Si}$ bridges and those appear at $967-950 \mathrm{~cm}^{-1}$ arise from $\mathrm{Si}-\mathrm{O}-\mathrm{Al}$ stretching vibrations. The imine $(\mathrm{C}=\mathrm{N})$ functional group of the ligand is clearly observed as intense and well-resolved bands at 1,632 and $1,629 \mathrm{~cm}^{-1}$ for immobilized complexes within the Al-MCM-41. The $\mathrm{C}=\mathrm{C}$ vibrations also appear at the regions 1,450 and $1,400 \mathrm{~cm}^{-1}$ (Fig. 4).

\subsection{UV-vis spectra studies}

The electronic spectra of Al-MCM-41, Fe(sal-L-his) $\mathrm{Cl} \bullet \mathrm{H}_{2} \mathrm{O}$ complex and $\mathrm{Fe}$ (sal-L-his) complex/Al-MCM-41 in ethanol are shown in Fig. 5. The spectrum of Fe(sal-Lhis)Cl complex in ethanol solution displays several bands at 216, 235, $255 \mathrm{~nm}$ (UV), and 312, 418-495 nm (visible), consistent with those reported before [29]. The first three bands are assigned to interligand transitions, characteristic of the Schiff base due to the salicylaldimine benzenoid $\pi-\pi^{*}$. The other observed near 312 and $420 \mathrm{~nm}$ bands in the absorption spectra must be LMCT [29, 37-39]. After immobilization of $\mathrm{Fe}$ (sal-L-his) $\mathrm{Cl}_{\bullet} \mathrm{H}_{2} \mathrm{O}$, these bands appear at the regions of $210,221,248,302,411$ and $501 \mathrm{~nm}$, respectively. Similar results were obtained for immobilized Fe(sal-L-his)(bpy) complex (Table 4).

\subsection{Magnetization studies}

In order to study the magnetic properties, the hysteresis loops of $\mathrm{Fe}$ (sal-L-his)complex and $\mathrm{Fe}$ (sal-L-his)(bpy) complexes were investigated at room temperature using vibrating sample magnetometry (VSM) and magnetization curves as shown in Fig. 6A, B, respectively. Based on the obtained results, both complex show superparamagnetic 
Table 1 Textural parameters, 'd' values, lattice parameters (100) and iron content of Al-MCM-41 samples

\begin{tabular}{lllllr}
\hline Samples & $\begin{array}{l}\mathrm{BET} \\
\left(\mathrm{m}^{2} \mathrm{~g}^{-1}\right)\end{array}$ & $\begin{array}{l}\text { Pore volume } \\
\left(\mathrm{mL} \mathrm{g}^{-1}\right)\end{array}$ & $\begin{array}{l}\text { Average pore } \\
\text { diameter }(\mathrm{nm})\end{array}$ & $\begin{array}{l}\mathrm{d} \text { value } \\
(\AA)\end{array}$ & $\begin{array}{l}\text { Lattice } \\
\text { parameter }(\AA)\end{array}$ \\
\hline Al-MCM-41 & 1,473 & 0.9601 & 2.053 & 37.57 & 43.38 \\
Fe(sal-L-his)complex/Al-MCM-41 & 1,214 & 1.091 & 2.140 & 39.06 & 45.10 \\
Fe(sal-L-his)(bpy) complex/Al-MCM-41 & 1,109 & 0.9623 & 2.143 & 38.43 & 44.37 \\
\hline
\end{tabular}

Table 2 Textural parameters, 'd' values and iron content of zeolite Y samples

\begin{tabular}{lllll}
\hline Material & $\begin{array}{l}\text { BET } \\
\left(\mathrm{m}^{2} \mathrm{~g}^{-1}\right)\end{array}$ & $\begin{array}{l}\text { Pore volume } \\
\left(\mathrm{mL} \mathrm{g}^{-1}\right)\end{array}$ & $\mathrm{d}$ value $(\AA)$ & $\mathrm{Fe}(\%)$ \\
\hline Zeolite Y & 596 & 0.03826 & 14.71 & 0 \\
Fe(sal-L-his)complex/zeolite Y & 549.3 & 0.04976 & 14.69 & 0.97 \\
Fe(sal-L-his)(bpy)complex/zeolite Y & 512.8 & 0.04594 & 14.64 & 0.71 \\
\hline
\end{tabular}

Table 3 FT-IR band assignments $\left(\mathrm{cm}^{-1}\right)$ for $\mathrm{Al}$ MCM-41 and Al-MCM-41 with immobilized complexes

\begin{tabular}{lllllll}
\hline Catalyst & $\mathrm{Si}-\mathrm{OH}$ & $(\mathrm{Si}-\mathrm{O}-\mathrm{Si})_{\text {asy }}$ & $(\mathrm{Si}-\mathrm{O}-\mathrm{Si})_{\text {as/sy }}$ & $(\mathrm{Si}-\mathrm{O}-\mathrm{Si})_{\mathrm{b}}$ & $\mathrm{C}=\mathrm{N}$ & $\mathrm{C}=\mathrm{C}$ \\
\hline Al-MCM-41 & 3,448 & 1,075 & 958,801 & 459 & - & - \\
Fe(sal-L-his)/Al-MCM-41 & 3,446 & 1,099 & 960,802 & 468 & 1,632 & 1,400 \\
Fe(sal-L-his)bpy/Al-MCM-41 & 3,432 & 1,077 & 966,801 & 461 & 1,629 & 1,450 \\
\hline
\end{tabular}

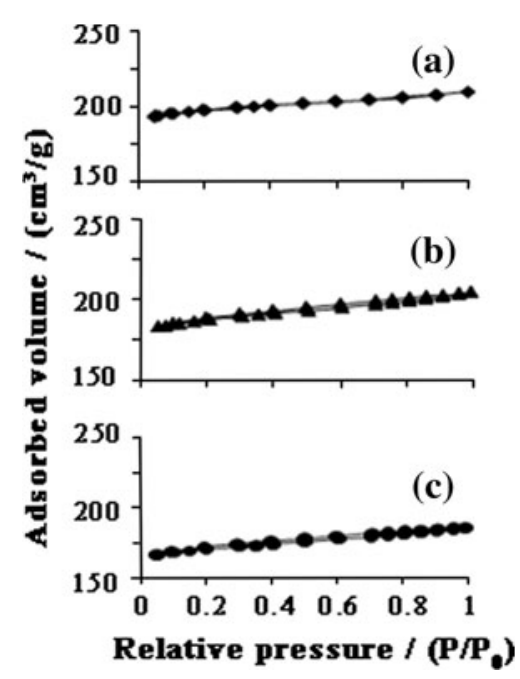

Fig. $4 \mathrm{~N}_{2}$ adsorption/desorption isotherms of $a$ zeolite $\mathrm{Y}, b \mathrm{Fe}$ (sal-Lhis)/zeolite $\mathrm{Y}$ and $c \mathrm{Fe}$ (sal-L-his)(bpy) complex/zeolite $\mathrm{Y}$

properties at low field although the Kapa of the former and the latter are 5.00 and 2.80, respectively. The consistency of these results with those of spin multiplicities obtained from theoretical calculations (see the supplementary) is notable [29].

\subsection{Reaction conditions}

The oxidation reactions were carried out in the presence of $\mathrm{Fe}$ (sal-L-his)complex/Al-MCM-41 as catalyst at different

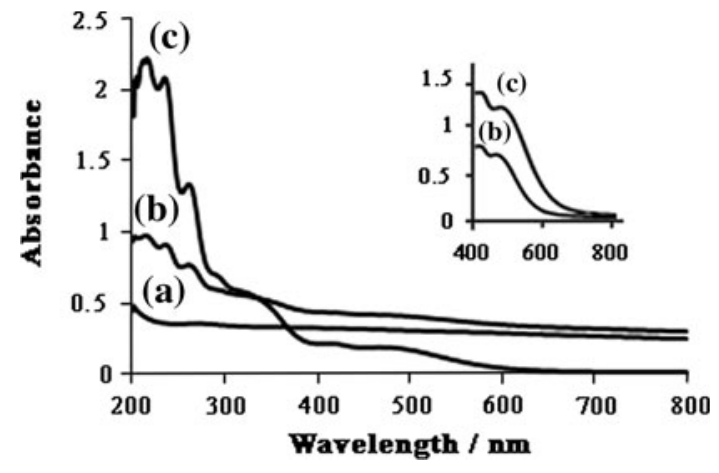

Fig. 5 The UV-vis spectra of $a$ Al-MCM-41, $b$ Fe(sal-L-his)complex/Al-MCM-41 and $c$ Fe(sal-L-his) $\mathrm{Cl}_{\bullet} \mathrm{H}_{2} \mathrm{O}$

times using cyclohexane as the representing substrate in order to choose the best reaction time. The results are presented in Fig. 7. As seen, cyclohexane is mostly oxidized within $6 \mathrm{~h}$, beyond which no considerable oxidation occurs during the next $4 \mathrm{~h}$. It was also found that using $0.1 \mathrm{~g}$ of catalyst is good enough for running the reactions when cyclohexane is a substrate (Fig. 8).

Oxidation results obtained for cyclohexane, methyl cyclohexane, cyclooctane and adamantane in $\mathrm{CH}_{3} \mathrm{CN}$ using $\mathrm{H}_{2} \mathrm{O}_{2}$ as oxidant in the presence of $0.1 \mathrm{~g}$ of $\mathrm{Fe}(\mathrm{sal}-\mathrm{L}-$ his)complex/zeolite Y, Fe(sal-L-his)(bipy)complex/zeolite Y, Fe(sal-L-his)complex/Al-MCM-41 and Fe(sal-L-his)bipy complex/Al-MCM-41 are given in Tables 5, 6, 7 and 8, respectively. Identification of the products was carried out 
Table 4 UV-vis data of iron complexes before and after immobilization within nanoreactors of Al-MCM-41

\begin{tabular}{llll}
\hline Compound & $\lambda_{\max }\left(\varepsilon, \mathrm{M}^{-1} \mathrm{~cm}^{-1}\right)$ in ethanol & & LMCT \\
\cline { 2 - 4 } & Interligand transition phenyl ring & $\pi \rightarrow \pi^{*}(\mathrm{C}=\mathrm{N})$ & $312(400), 420(200), 480(150)$ \\
$\mathrm{Fe}\left(\right.$ sal-L-his)Cl•H $\mathrm{H}_{2} \mathrm{O}$ & $216(1,466), 235(1,400)$ & $255(913)$ & $302,411,468$ \\
$\mathrm{Fe}($ sal-L-his)/Al-MCM-41 & 210,221 & 248 & $338(375), 501(187)$ \\
$\mathrm{Fe}($ sal-L-his)bpyCl & $204(2,562), 234(2,125)$ & $284(2,000)$ & 332,495 \\
$\mathrm{Fe}($ sal-L-his)bpy/Al-MCM-41 & 200,226 & 256 & \\
\hline
\end{tabular}

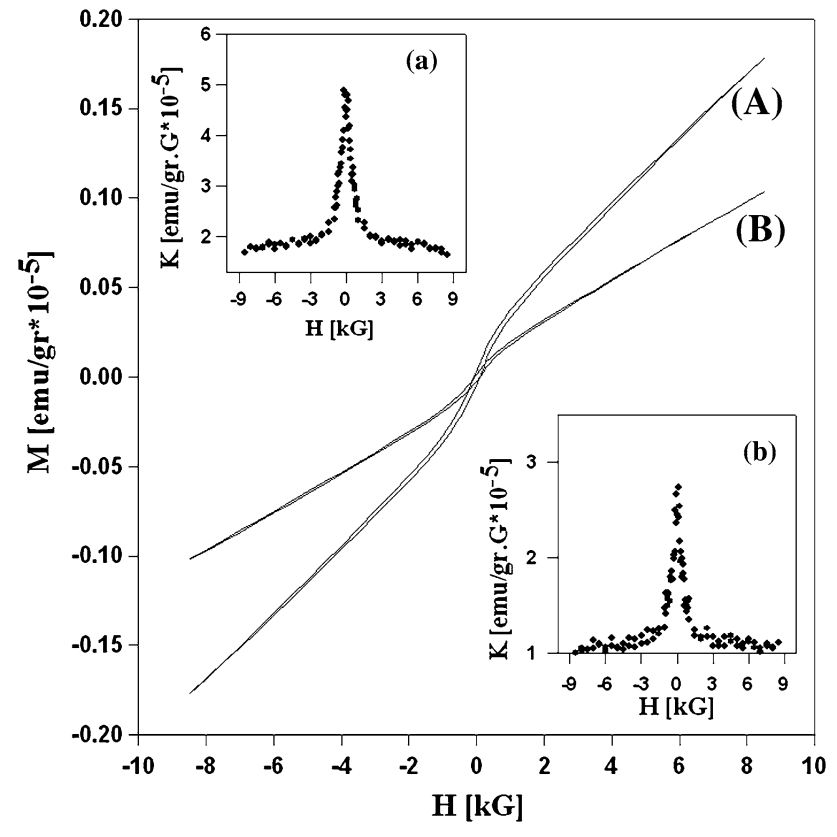

Fig. 6 Magnetization curve of $A \mathrm{Fe}$ (sal-L-his)complex and $B \mathrm{Fe}$ (salL-his)(bpy)complex, inset Kapa versus magnetic field for $a \mathrm{Fe}$ (sal-Lhis)complex and $b \mathrm{Fe}$ (sal-L-his)(bpy)complex has been reported

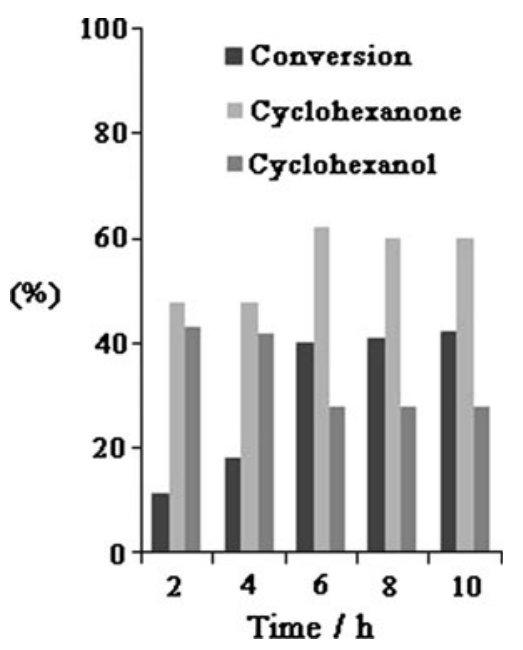

Fig. 7 Effect of time on cyclohexane oxidation with $\mathrm{H}_{2} \mathrm{O}_{2}$ catalyzed by [Fe(sal-L-his)complex/Al-MCM-41

by comparison of the GC-Mass spectra with those of the authentic samples. As expected, all catalysts are active with about $47-100 \%$ conversion. We have included the effect

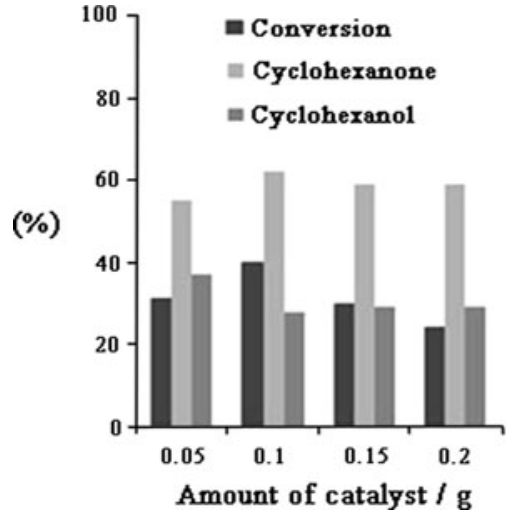

Fig. 8 Effect of the amount of Fe(sal-L-his)complex/Al-MCM-41 on cyclohexane conversion

of [ $\mathrm{Fe}$ (sal-L-his) $\left.\mathrm{ClH}_{2} \mathrm{O}\right] \bullet 3 \mathrm{H}_{2} \mathrm{O}$ and [ $\mathrm{Fe}$ (Sal-L-his)(bipy)Cl] as homogeneous catalysts in Table 5 in order to make the comparisons more convenient. It was also found that no significant conversion of cyclohexane was observed with Al-MCM-41 and zeolite $\mathrm{Y}$ void of complexes. The catalytic activity of the recovered Fe(sal-L-his)/Al-MCM-41was similar to that of the fresh catalyst. Notably, no desorption was observed during the course of reactions. Particularly significant is the effect of $\mathrm{Fe}$ (sal-L-his)(bpy)complex/Al-MCM-41 as catalyst on cyclooctane oxidation, with $90 \%$ conversion and $85 \%$ selectivity toward the formation of cyclooctanone as the major product (Table 7). The oxidation results of adamantane to adamantane-1-ol, and adamantane-2-one are presented in Table 8 . The maximum amount of conversion and selectivity occurs in the presence of Fe(sal-L-his)(bpy)complex/Al-MCM-41 as catalyst (Table 8, entry 20). Compared to other substrates, oxidation reaction of adamantane proceeds less efficiently (98\% conversion within $24 \mathrm{~h}$ ).

The stability of the Fe(sal-L-his)(bpy)complex/Al-MCM41 was also studied by recycling the recovered catalyst and determination of the metal content using atomic absorption spectroscopy. The iron content was shown to be $0.755 \%$ with very little change $(0.005 \%$,) with respect to the initial catalyst iron content $(0.760 \%)$. As shown in Table 5, entry 8, the recovered Fe(sal-L-his)(bpy)complex/Al-MCM-41 catalyzed the oxidation of cyclohexane with a minor decrease in conversion although with the same selectivity toward the formation of the corresponding oxides. 
Table 5 Results of cyclohexane oxidation with $\mathrm{H}_{2} \mathrm{O}_{2}$ in the presence of iron catalysts

\begin{tabular}{|c|c|c|c|c|c|c|}
\hline Entry & Catalyst & Conversion $(\%)$ & $\mathrm{Cy}-\mathrm{OH}^{\mathrm{a}}(\%)$ & $\mathrm{Cy}=\mathrm{O}^{\mathrm{b}}(\%)$ & Others $^{\mathrm{c}}(\%)$ & TON \\
\hline 1 & $\mathrm{Fe}$ (sal-L-his)complex/zeolite Y & 65 & 30 & 60 & 10 & 751 \\
\hline 2 & $\mathrm{Fe}$ (sal-L-his)complex/Al-MCM-41 & 69 & 25 & 63 & 12 & 722 \\
\hline 3 & Fe(sal-L-his)(bpy)complex/zeolite Y & 92 & 28 & 63 & 9 & 1,460 \\
\hline 4 & Fe(sal-L-his)(bpy)complex/Al-MCM-41 & 100 & 27 & 63 & 10 & 1,481 \\
\hline 5 & {$\left[\mathrm{Fe}(\right.$ sal-L-his $\left.) \mathrm{Cl} \bullet \mathrm{H}_{2} \mathrm{O}\right]$} & 47 & 46 & 54 & - & 543 \\
\hline 6 & {$[\mathrm{Fe}($ sal-L-his)(bpy)Cl] } & 55 & 30 & 70 & - & 873 \\
\hline 7 & $\mathrm{Fe}$ (sal-L-his)complex/zeolite $\mathrm{Y}^{\mathrm{d}}$ & 61 & 24 & 63 & 12 & 639 \\
\hline 8 & $\mathrm{Fe}$ (sal-L-his)(bpy)complex/Al-MCM- $41^{\mathrm{e}}$ & 95 & 26 & 64 & 10 & 1,400 \\
\hline
\end{tabular}

Reaction conditions: catalyst $(0.1)$, substrate $(20 \mathrm{mmol}), \mathrm{H}_{2} \mathrm{O}_{2}(30 \%, 36 \mathrm{mmol})$, solvent $\left(\mathrm{CH}_{3} \mathrm{CN}, 5 \mathrm{~mL}\right.$, under reflux), time $(6 \mathrm{~h})$, TON is the $\mathrm{mmol}$ of products to the $\mathrm{mmol}$ of the iron present in catalyst

${ }^{a} \mathrm{Cy}-\mathrm{OH}$; cyclohexanol

b $\mathrm{Cy}=\mathrm{O}$; cyclohexanone

c Others as minor products are 4-hydroxy cyclohexanone and 1,4-cyclohexanedione

d, e Reusability results

Table 6 Results of methyl cyclohexane oxidation with $\mathrm{H}_{2} \mathrm{O}_{2}$ in the presence of iron catalysts

\begin{tabular}{|c|c|c|c|c|c|}
\hline Entry & Catalyst & Conversion $(\%)$ & $\mathrm{Me}-\mathrm{Cy}-\mathrm{OH}^{\mathrm{a}}(\%)$ & $\mathrm{Me}-\mathrm{Cy}=\mathrm{O}^{\mathrm{b}}(\%)$ & TON \\
\hline 9 & $\mathrm{Fe}$ (sal-L-his)complex/zeolite Y & 31 & 38 & 62 & 358 \\
\hline 10 & Fe(sal-L-his)complex/Al-MCM-41 & 40 & 34 & 66 & 418 \\
\hline 11 & Fe(sal-L-his)(bpy)complex/zeolite Y & 51 & 19 & 81 & 809 \\
\hline 12 & $\mathrm{Fe}$ (sal-L-his)(bpy)complex/Al-MCM-41 & 57 & 20 & 80 & 840 \\
\hline
\end{tabular}

Reaction conditions are similar to Table 5

${ }^{a}$ Methyl cyclohexanol

b Methyl cyclohexanone

Table 7 Results of cyclooctane oxidation with $\mathrm{H}_{2} \mathrm{O}_{2}(30 \%)$ in the presence of iron catalysts

\begin{tabular}{|c|c|c|c|c|c|c|}
\hline Entry & Catalyst & Conversion $(\%)$ & $\mathrm{Cy}-\mathrm{Ol}^{\mathrm{a}}(\%)$ & $\mathrm{Cy}=\mathrm{O}^{\mathrm{b}}(\%)$ & Others $^{\mathrm{c}}(\%)$ & TON \\
\hline 13 & $\mathrm{Fe}$ (sal-L-his)complex/zeolite Y & 51 & 10 & 60 & 9 & 589 \\
\hline 14 & Fe(sal-L-his)complex/Al-MCM-41 & 68 & 9 & 63 & 5 & 712 \\
\hline 15 & $\mathrm{Fe}$ (sal-L-his)(bpy)complex/zeolite $\mathrm{Y}$ & 69 & 9 & 82 & 5 & 1,095 \\
\hline 16 & Fe(sal-L-his)(bpy)complex/Al-MCM-41 & 90 & 15 & 85 & 0 & 1,075 \\
\hline
\end{tabular}

${ }^{\text {a }}$ Cyclooctanol

b Cyclooctanone

c Unidentified

The final note worthwhile to be emphasize is the high heterogeneous character of the Fe(sal-L-his)(bpy)complex/ Al-MCM-41catalyst used in this work. The experimental evidences ruled out the participation of any leached active species from the solid catalysts in the oxidation reactions.

\subsection{Mechanism of catalytic activity}

Cyclohexane oxidation using $\mathrm{H}_{2} \mathrm{O}_{2}$ as oxidant seems to proceed via a radical mechanism initiating from transition metal catalyzed decomposition of $\mathrm{H}_{2} \mathrm{O}_{2}$. It is generally accepted that $\mathrm{Fe}^{\mathrm{IV}}=\mathrm{O}$ and $\mathrm{HO}$ or $\mathrm{HOO}$ radicals can be generated via the reaction between hydroperoxide and the ferric ion (Scheme 2, Eqs. 2a and 2b) [40-43]. The key radical intermediates are obtained through the radical chain reactions (Scheme 2, Eqs. 3, 4 and 5). Cycloxexanol and cyclohexanone are generated via the homolytic (Eq. 6) and heterolytic (Eq. 7) decompositions, respectively. Cyclohexanol and cyclohexanone can also be produced via a Russell termination reaction [44] (Eq. 8).

Compared to Fe(sal-L-his)complex/Al-MCM-41 (Table 5, entry 2), the results presented in Table 5 clearly indicate the enhanced catalytic activity of Fe(sal-L-his)(bpy)complex/AlMCM-41 (entry 4). The oxidation step (Eq. 2a) is generally 
Table 8 Results of adamantane oxidation with $\mathrm{H}_{2} \mathrm{O}_{2}$ in the presence of iron catalysts

\begin{tabular}{|c|c|c|c|c|c|c|}
\hline Entry & Catalyst & Conversion $(\%)$ & $\mathrm{Ad}-\mathrm{OH}^{\mathrm{a}}(\%)$ & $\mathrm{Ad}=\mathrm{O}^{\mathrm{b}}(\%)$ & TON & $\mathrm{C}^{3}-\mathrm{H} / \mathrm{C}^{2}-\mathrm{H}^{\mathrm{c}}$ \\
\hline 17 & Fe(sal-L-his)complex/zeolite Y & 73 & 27 & 73 & 42 & 1.11 \\
\hline 18 & Fe(sal-L-his)complex/Al-MCM-41 & 82 & 28 & 72 & 65 & 1.17 \\
\hline 19 & $\mathrm{Fe}$ (sal-L-his)(bpy)complex/zeolite Y & 83 & 28 & 72 & 44 & 1.17 \\
\hline 20 & Fe(sal-L-his)(bpy)complex/Al-MCM-41 & 98 & 30 & 70 & 70 & 1.29 \\
\hline
\end{tabular}

Reaction conditions: catalyst $(0.1 \mathrm{~g})$, substrate $(1 \mathrm{mmol}), \mathrm{H}_{2} \mathrm{O}_{2}(30 \%, 25 \mathrm{~mL})$, solvent $\left(\mathrm{CH}_{3} \mathrm{CN}, 15 \mathrm{~mL}\right.$, under reflux $)$, time (24 h), TON is the $\mathrm{mmol}$ of products to the mmol of the iron present in catalyst

a Adamantane-1-ol

b Adamantane-2-one

c The selectivity parameter defined by the relative reactivity of tertiary $\mathrm{C}-\mathrm{H}$ to secondary $\mathrm{C}-\mathrm{H}$ bonds $=(\% \mathrm{Ad}-\mathrm{OH} / 4) /(\% \mathrm{Ad}=\mathrm{O} / 12)$

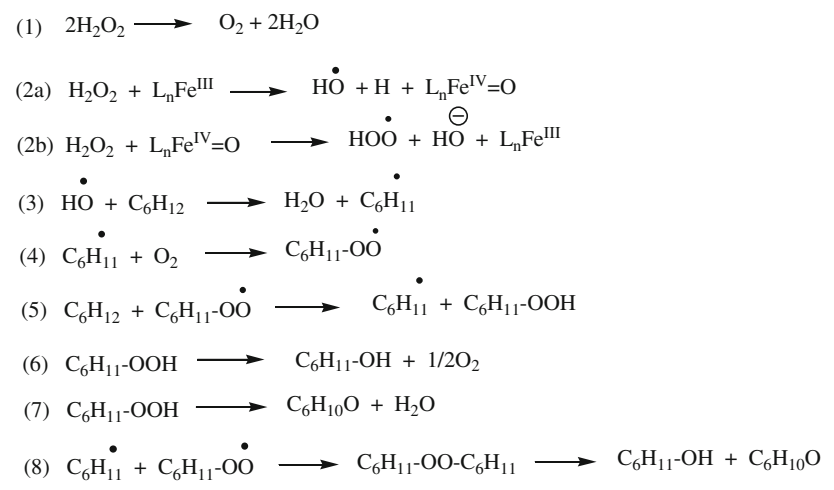

Scheme 2 Suggested mechanism for the oxidation of cyclohexane

assumed to be facilitated by suitable ligands capable of stabilizing the hypervalent state of the iron-oxo species by resonance. Since Collman and co-workers have reported that oxygen-radical $\mathrm{Ph}_{2} \mathrm{NH}$ inhibits an oxidation [45], we used it to have insight into the reaction mechanism. Using an equimolar of trapping agent completely suppressed cyclohexane oxidation based on the GC analysis of the product mixture, which showed that cyclohexane has been left intact.

\section{Conclusions}

The iron Schiff base complexes immobilized within of AlMCM-41 and zeolite Y designated as Fe(sal-L-his)complex/ Al-MCM-41, Fe(sal-L-his)complex/zeolite Y, Fe(sal-L-his) (bipy)complex/Al-MCM-41and Fe(sal-L-his)(bipy)complex/ zeolite $\mathrm{Y}$, were prepared and characterized. It was found that Fe(sal-L-his)(bipy)complex/Al-MCM-41 successfully catalyze the oxidation of cyclooctane to cyclooctanol and cyclooctanone with $90 \%$ conversion and $85 \%$ selectivity toward the formation of ketone. Similarly, cyclohexanol and cyclohexanone were identified as the major products in the oxidation of cyclohexane. When methyl cyclohexane is used as substrate only methyl cyclohexanol and methyl cyclohexanone are formed. When adamantine is as substrate, the oxidation process is slow relative to the other substrate. These points together with utilization of $\mathrm{H}_{2} \mathrm{O}_{2}$ as green oxidant and observation of no metal desorption during the course of reactions are the highlights of this presentation.

Acknowledgments This work was financially supported by the University of Alzahra.

\section{References}

1. R.G. Sheldon, B.J. Kochi, Metal-Catalyzed Oxidations of Organic Compounds (Academic Press, New York, 1981)

2. A.E. Shilov, G.B. Sulphin, Chem. Rev. 97, 2879 (1997)

3. M.J. Gunter, P. Turner, Coord. Chem. Rev. 108, 115 (1991)

4. B. Meunier, S.P. De Viesser, S. Shaika, Chem. Rev. 104, 3947 (2004)

5. T. Katsuki, Chem. Soc. Rev. 33, 437 (2004)

6. C.A. Root, J.D. Hoeschele, C.R. Cornman, J.W. Kampf, V.I. Pecoraro, Inorg. Chem. 32, 3855 (1993)

7. M. Klopstra, R. Hage, R.M. Kellogg, B.L. Feringa, Tetrahedron Lett. 44, 4581 (2003)

8. D.C. Sherrington, Catal. Today $\mathbf{5 7 , 8 7}$ (2000)

9. H. Fujii, Coord. Chem. Rev. 226, 51 (2002)

10. I. Bertini, H.B. Gray, S.J. Lippard, J.S. Valentine, Bioinorganic Chemistry, ch. 5 (University Science Books, Mill Valley, 1994)

11. G.C. Silva, G.L. Parrilha, N.M.F. Carvahlo, V. Drago, C. Fernandes, A. Hom, O.A.C. Antunes, Catal. Today 133, 684 (2008)

12. N.M.F. Carvalho, A. Hom, O.A.C. Antunes, Appl. Catal. A: Gen. 305, 140 (2006)

13. J. England, G.J.P. Britovsek, N. Rabadia, A.J.P. White, Inorg. Chem. 46, 3752 (2007)

14. S. Nayak, P. Gamez, B. Kozlevcar, A. Pevec, O. Roubeau, S. Dehnen, J. Reedijk, Polyhedron 29, 2291 (2010)

15. S.A. Abdel-Latif, H.B. Hassib, Y.M. Issa, Spectrochimica Acta Part A 67, 950 (2007)

16. A. Bottcher, M.W. Grinsstaff, J.A. Labinger, H.B. Gray, J. Mol. Catal. A: Chem. 117, 229 (1997)

17. M.W. Grinsstaff, M.G. Hill, Labinger, H.B. Gray, Science 264, $1311(1994)$

18. K.C. Gupta, S.A. Kumar, C-.C. Lin, Coord. Chem. Rev. 253, 1926 (2009)

19. D.E. De Vos, M. Dams, B.F. Seis, P.A. Jacobs, Chem. Rev. 102, 3615 (2002)

20. S. Jana, B. Dutta, R. Bera, S. Koner, Langmuir 23, 2492 (2007)

21. P.K. Saha, B. Dutta, S. Jana, R. Bera, S. Saha, K.i Okamoto, S. Koner, Polyhedron 26, 563 (2007) 
22. B. Dutta, S. Jana, R. Bera, P. Kumar Saha, S. Koner, Appl. Catal. A 318, 89 (2007)

23. R.J. Correa, G.C. Salomao, M.H.N. Olsen, F.L. Cardozo, V. Drago, C. Fernandes, O.A.C. Antunes, Appl. Catal. A: Gen. 366, 35 (2008)

24. I.L. Viana, C.M. Rosa, C.p Manso, O.A. Serra, Y. Iamamoto, J. Mol. Catal. A: Chem. 160, 199 (2000)

25. A.B. Sorokin, S. Magnematine, C. Pergale, J. Mol. Catal. A: Chem. 182, 267 (2002)

26. F. Farzaneh, M. Poorkhosravani, M. Ghandi, J. Mol. Catal. A: Chem. 308, 108 (2009)

27. S.V. Sirotin, A.Y. Tolbin, I.F. Moskovaskaya, S.S. Abramchuk, L.G. Tomilova, B.V. Romanovsky, J. Mol. Catal. A: Chem. 319, 39 (2010)

28. M.A. Zanjanchi, S. Asgari, Solid State Ionics 171, 277 (2004)

29. L. Casella, M. Gullotti, A. Pintar, L. Messori, A. Rockenbauer, M. Gyorl, Inorg. Chem. 26, 1031 (1987)

30. H. Hosseini Monfared, S. Sadighian, M.A. Kamyabia, P. Mayer, J. Mol. Catal. A: Chem. 304, 139 (2009)

31. M. Bagherzadeh, M. Amini, Inorg. Chem. Commun. 12, 21 (2009)

32. M. Selvaraj, A. Pandurangan, K.S. Seshadri, P.K. Sinha, V. Krishnasamy, K.B. Lal, J. Mol. Catal. A: Chem. 192, 153 (2003)

33. J. Dzierzak, M. Lefenfeld, R. Raja, Top. Catal. 52, 1669 (2009)
34. M. Masteri-Farahani, F. Farzaneh, M. Ghandi, Catal. Commun. 8, 6 (2007)

35. J. Lynch, Physico-Chemical Analysis of Industrial Catalysts, ch. 1 (Technip ed., Paris, 2001)

36. T.A. Fernandes, C.D. Nunes, P.D. Vaz, M.J. Calhorda, P. Brandao, J. Rocha, I.S. Goncalves, A.A. Valente, L.P. Ferreira, M. Godinho, P. Ferreira, Micropor. Mesopor. Mater. 112, 14 (2008)

37. H.S. Abbo, J.J. Titinchi, R. Prasad, S. Chand, J. Mol. Catal. A: Chem. 225, 225 (2005)

38. A.A.A. Emara, A.A.A. Abou-Hussen, Spectrochimica Acta Part A 64, 1010 (2006)

39. K.M. Parida, M. Sahoo, S. Singha, J. Mol. Catal. A: Chem. 329, 7 (2010)

40. F. Gozzo, J. Mol. Catal. A: Chem. 171, 1 (2001)

41. Z. Wangcheng, L. Guanzhong, G. Yanglong, G. Yun, W. Yanqin, W. Yunsong, Z. Zhigang, L. Xiaohui, J. Rare Earths 26, 515 (2008)

42. R. Kumar, S. Sithambarama, S.L. Suib, J. Catal. 262, 304 (2009)

43. I. Hermans, P.A. Jacobs, J. Peeters, J. Mol. Catal. A: Chem. 251, 221 (2006)

44. G.A. Russell, J. Am. Chem. Soc. 79, 3871 (1957)

45. L.M. Slaughter, J.P. Collman, T.A. Eberspacher, J.R. Brauman, Inorg. Chem. 43, 203 (2004) 